\title{
INTELLIGENT PRODUCTS: INTELLIGENT BEINGS OR AGENTS?
}

\author{
Paul Valckenaers and Hendrik Van Brussel \\ K.U.Leuven, Mechanical Engineering, Belgium \\ Paul.Valckenaers@mech.kuleuven.be
}

\begin{abstract}
This paper introduces a novel concept, the intelligent being, as a vehicle to achieve suitability for integration. The paper argues for a role of the intelligent being that is analogous to what maps contribute in navigation systems (and may become as important). The concept is applied to intelligent product instances, intelligent product types and intelligent resources alike.
\end{abstract}

\section{INTRODUCTION}

This paper focuses on suitability for integration. It identifies which components and subsystems can be designed such that they are highly suited for integration. And, the paper discusses how to design such components. The motivation to present this research in an intelligent product context resides in its aptness as a foundation for components that are suited for integration.

The quest for system components suited for integration dates back to the CIM era and its "islands of automation." The disappointing performance of those early-day integration efforts triggered a search for the root causes. Thus, research in Intelligent Manufacturing Systems has been looking at the fundamental science of the artificial, addressing complex adaptive systems, holonic systems, etc. A major insight is that manmade system components incorporate varying degrees of choice, and that conflicting choices are a necessary condition for integration problems.

Intelligent beings avoid these choices - at least in their application domain, not in the ICT dimensions. The full set of these in-depth insights distinguishes more levels and nuances (e.g. inertia build-up by these choices) but the intelligent being concept covers the most important part from a practical point of view. It is a clean concept delivering hard integrate-ability guarantees. The intelligent being inherits those guarantees from the real world, which it reflects.

This paper first discusses intelligent products as a composition of an intelligent being and an intelligent agent. Next, it addresses the intelligent products architecture. This includes a list of services offered by the intelligent being within the intelligent product. Finally, conclusions are given. 


\section{INTELLIGENT PRODUCTS}

This section identifies what intelligence may be added to a product without losing the ability to integrate the resulting intelligent product into any overall system. It also introduces the concept of an intelligent being.

\subsection{Products are "beings"}

Since the 1980s, software engineering recognizes that, to create long-lived information systems, the foundations of a system have to reflect the corresponding reality (Jackson, 1995). The design of an intelligent product - whose main function is to reflect a real product - naturally applies and exploits this principle.

The real world possesses a much sought-after property by software and system designers: it is a (very large) coherent and consistent collection of components and subsystems. This is the reason why the above software engineering principle is so effective. Software components reflecting reality are protected by this reality. Any design conflict involving such a protected component simultaneously has a conflict with reality. Consequently, solutions of such conflict leave these reality-reflecting software components intact (Valckenaers, 2003).

The oldest information artifacts that reflect some part of reality are maps. Maps add intelligence to reality. Maps are top-performing artifacts concerning integration issues. People have no problems using multiple maps even if they overlap geographically or deliver overlapping information (e.g. a tourist map, navigation map and topological map of the same area). Conflicts between maps indicate erroneous implementations and are easily corrected by checking with the corresponding reality.

For software designers, this may seem utopia. Indeed, a complete functional software system cannot be developed through exclusively reflecting reality such that this reality protects all the software components as effectively as it protects maps. However, navigation software hints that a major part of the software system may benefit from such protection in applications that are closely connected to parts of the real world. Intelligent products certainly are suitable candidates.

\subsection{Intelligent Beings}

Intelligent Beings are software components that

1. are sufficiently sophisticated to be considered intelligent and

2. reflect some part of reality in a choice-free manner that offers protection as discussed above

Actually, these intelligent software artifacts emphasize and mirror existence (being) in their world-of-interest. In contrast, intelligent agents emphasize action and decisionmaking on behalf of such an existence in their world-of-interest (analogous to the relation between an artist and his/her agent).

To illustrate the distinction between the protection enjoyed by an intelligent being and the exposure endured by intelligent agents, the following story may help. It is the actual transcript of a US naval ship with Canadian authorities off the coast of Newfoundland in October, 1995. This radio conversation was released by the Chief of Naval Operations on 10-10-95: 
- Canadians: "Please divert your course $15^{\circ}$ to the South to avoid a collision."

- Americans: "Please divert your course $15^{\circ}$ to the North to avoid a collision."

- Canadians: "We Repeat. Recommend you divert YOUR course 15 degrees to the South to avoid a collision."

- Americans: "This is the captain of a US Navy ship. I say again, divert YOUR course."

- Canadians: "No, I say again, you divert YOUR course."

- Americans: "THIS IS THE AIRCRAFT CARRIER USS ABRAHAM LINCOLN, THE SECOND LARGEST SHIP IN THE UNITED STATES' ATLANTIC FLEET. WE ARE ACCOMPANIED BY THREE DESTROYERS, THREE CRUISERS AND NUMEROUS SUPPORT VESSELS. I DEMAND THAT YOU CHANGE YOUR COURSE 15 DEGREES NORTH. THAT'S ONE-FIVE DEGREES NORTH, OR COUNTER MEASURES WILL BE UNDERTAKEN TO ENSURE THE SAFETY OF THIS SHIP."

- Canadians: "This is a lighthouse. Your call."

The Canadians are enjoying the role/situation of an intelligent being. The Americans are enduring the role/situation of an intelligent agent, erroneously interacting with the intelligent being as if it were an agent.

What the above story illustrates is that in conflicts, the solution cannot be expected from adaptations of the intelligent being, especially when the intelligent being is able to track its corresponding reality. Solutions only involve the intelligent agents and reality itself. Integration and collaboration issues affecting the intelligent being in the story are limited to the radio conversation itself: frequency, modulation, language and jargon spoken, etc. These are minor compared to the real-world stakes.

\subsection{Intelligent product $=$ "intelligent being + intelligent agent"}

An intelligent product will be a combination of an intelligent being and an intelligent agent. The intelligent being is restricted to functionality and services for which the corresponding reality provides adequate protection. This requirement cannot be compromised in any way since the intelligent being would loose its most attractive property, which it shares with old-fashioned maps. Any functionality or service that requires decision-making, not covered by reality, is delegated to the intelligent agent (cf. figure 1).

In nature (i.e. in humans and animals), the functionality of the intelligent agent is included within the intelligent being. Such implementation of multiple functions in a single embodiment is characteristic for natural systems. For instance, birds combine lift and propulsion in their flapping wings. In contrast, successful manmade artifacts often have separate embodiments for every main function. Aircraft have fixed wings for lift that are separate from the engines providing propulsion. A key motivation for this separation in artificial systems is specialization in the design and production of these components (separate organizations are responsible). Separate embodiments effectively serve much larger user groups over longer periods of time than a single combined embodiment.

For the intelligent being and intelligent agent, this motivation most strongly applies since they have radically different domains - concerning life cycle, location and service type - across which they remain functional. In fact, the intelligent being is functional whenever its reflected reality is. In contrast, the intelligent agent is functional wherever its decision-making capabilities provide adequate service. Techno-economic pressures call for two separate embodiments. In comparison, a hardwired combination of an intelligent agent and its intelligent being would lack critical user mass and would be short-lived. 
The stability and suitability for integration of intelligent beings makes them the prime candidates for the foundation of the overall system. Therefore, the following section discusses what services and functionality can be delivered by intelligent beings within intelligent products.

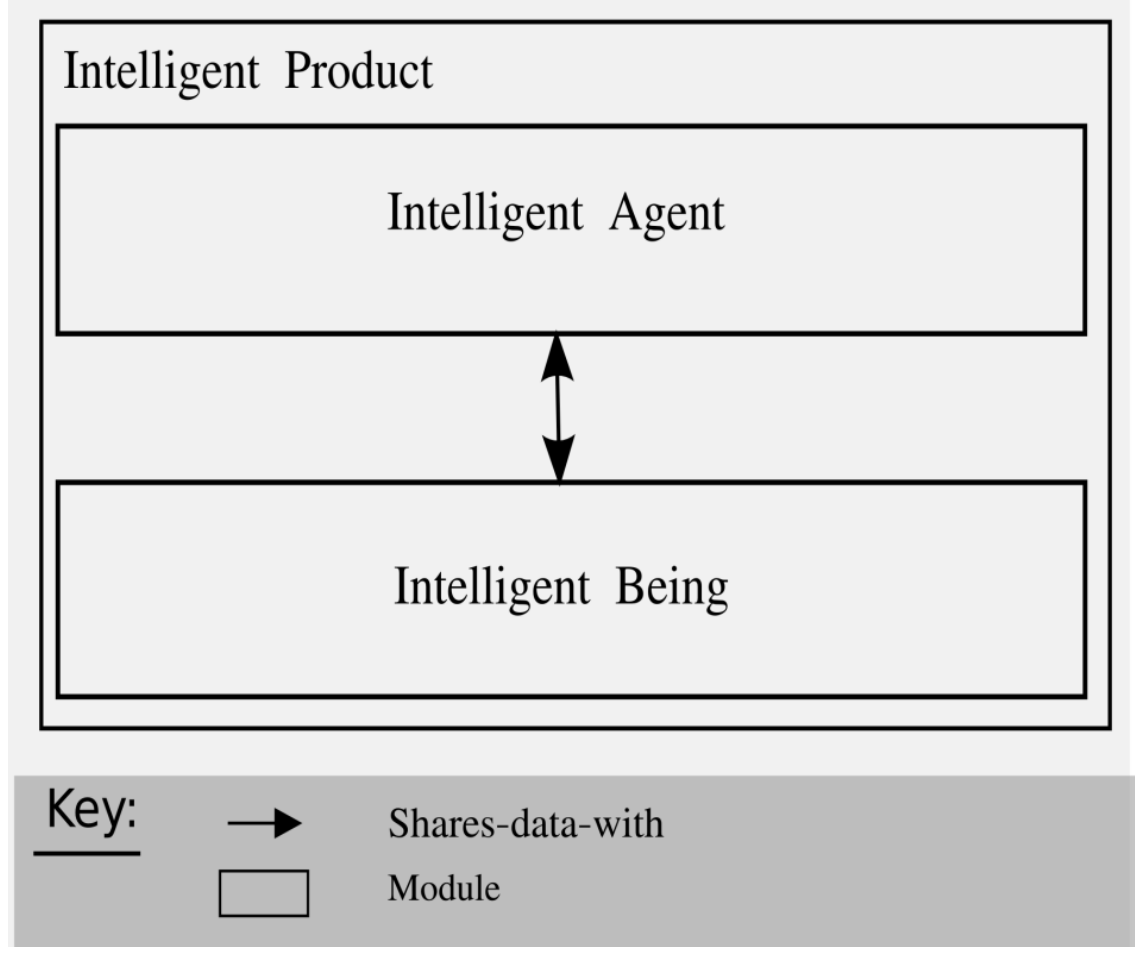

Figure 1. Intelligent product architecture

\section{INTELLIGENT PRODUCTS ARCHITECTURE}

An intelligent product has two main modules: the intelligent being and the intelligent agent. The intelligent agent performs all decision-making tasks. It accesses the real world exclusively through the intelligent being. In environments for multi-agent systems, as discussed in (Valckenaers, 2007), the agents interact with the real world through environment entities. Intelligent beings are prime candidates for this environment entity role (cf. figure 2). The intelligent being reflects a real-world entity, while delegating all decision-making to the intelligent agent. 


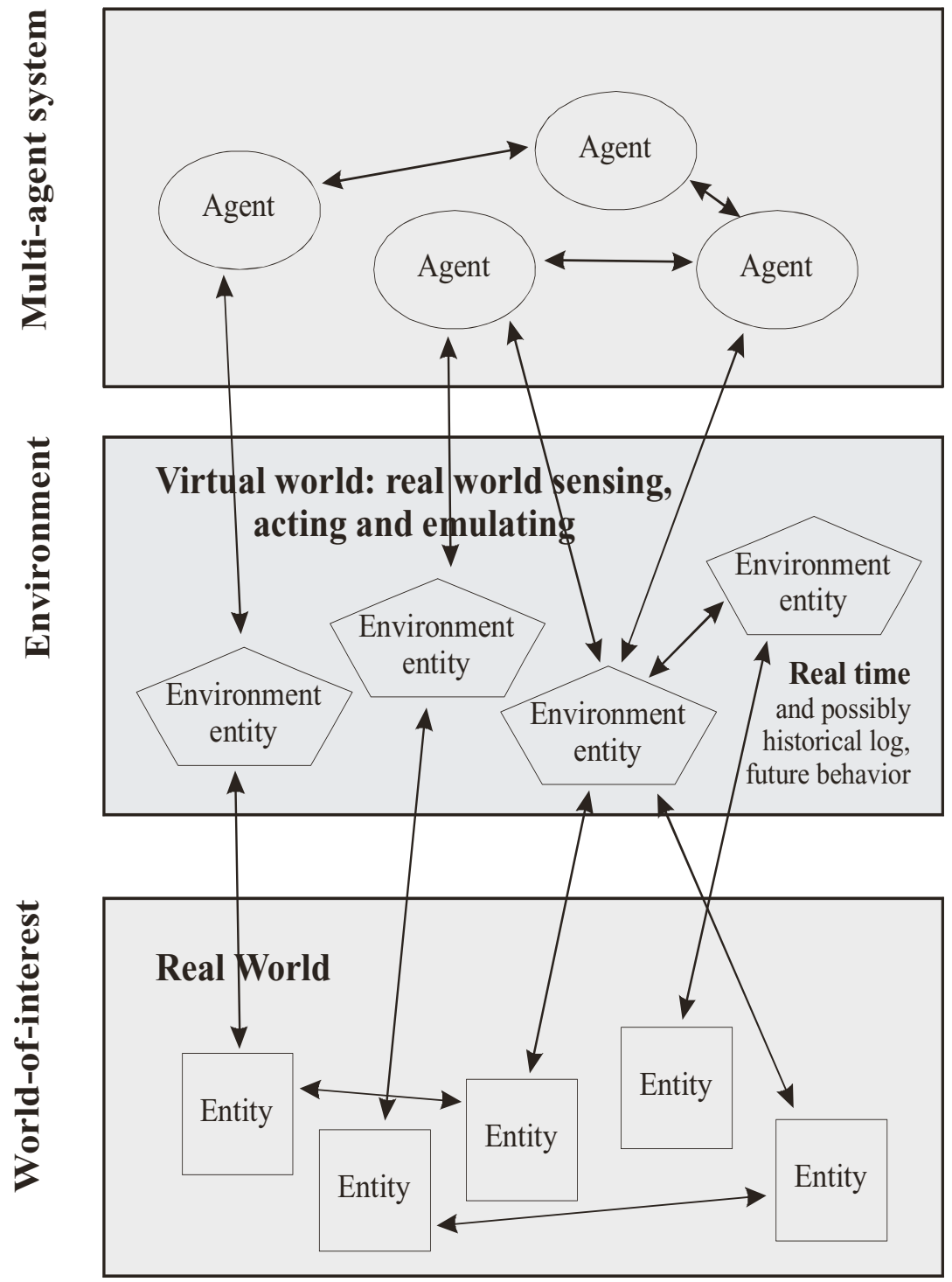

Figure 2. Mirroring the real world in physical-world-rooted applications

An intelligent agent exchanges information with its corresponding intelligent being. The agent can perform actions in the environment (for instance, the agent can request actuation of the physical machine). The agent can also perceive the intelligent being (for instance, it can observe its capabilities or check if a certain task can be performed on the machine the intelligent being reflects).

Intelligent beings may offer decision-free reality-reflecting services belonging to the following categories:

- Self-models (static). The intelligent being informs about product properties (technical specifications). It is knowledgeable about the current state of the product instance and the possible states of instances of its product type. 
- Self-models (dynamic). The intelligent being provides information on how the product evolves or may evolve over time. It is knowledgeable about the possible transitions between possible states and the properties of these transitions.

- Emulation. The intelligent being supports emulation of its real-world counterpart that, among others, can be used for plan verification.

- Acquaintances network. The intelligent being is able to contact its surrounding entities and to provide their contact coordinates to virtual visitors. This includes the resources on which the product resides, its owner, its customer, its intelligent agent, and the links to its real-world counterpart. This feature creates a network across which intelligent agents and beings may travel virtually.

- Stigmergie. Intelligent beings offer the cyber-counterpart of tagging (post-its) in the real world. This permits virtual visitors to deposit, observe and modify information attached to the intelligent being. A refresh-or-forget mechanism may be applied to cope with a dynamic environment.

- Access to the real world. Raw sensor data needs processing (filtering, aggregation, fusing, interpreting) before it provides useful information. Moreover, sensor data often is only available at given instances and state estimators need to compensate (resulting in virtual sensors). In the reverse direction, raw actuating needs encapsulating by proper services. The intelligent being provides this service while delegating possible choices to its agent.

- Traceability. The intelligent being provides tracking and tracing. It provides access to information about the current state of the product instance as well as the trajectory leading to this state. The intelligent product may provide a high-quality time base against which the trace is recorded.

- Forecasting (reflecting agent intentions). The emulation/modeling services provided by intelligent product support some simple forecasting. For instance, the models may indicate how a heat treatment is likely to affect product properties. To move beyond this, the intelligent being extends the part of reality that it reflects. It extends this part of reality to include the known intentions and commitments of agents that affect its short-term future. This produces a short-term forecast for the product itinerary and a load forecast for the resources involved. Valckenaers (2005) discusses a Holonic Manufacturing Execution System in which intelligent products offer such forecasting service. Parunak (2007) uses a similar principle to generate short-term forecasts in a self-organizing fashion. Both designs produce predictions for resources loads and user/product trajectories. Note that the intelligent beings only need agents that are capable to take decisions in the actual situations in order to generate forecasts. Agents must not possess models themselves to produce forecasts.

These responsibilities reflect the experience of the authors in building intelligent beings. Variations on this architecture, depending on the requirements of the application, are possible. Importantly, the functionality provided by an intelligent being can be expanded and enhanced without causing an avalanche of software maintenance or validation efforts. This situation is analogous to enhancing maps, indicating perhaps where the one-way streets are, and expanding maps to cover maybe a wider geographic area. This does not necessarily apply to the associated intelligent agents. Indeed, a more accurate and detailed representation of reality often renders a decision mechanism inadequate. Intrinsically, intelligent beings (inside intelligent products) are maintenancefree until the corresponding reality changes in a manner that the intelligent beings are unable to track. 


\section{CONCLUDING REMARKS}

This paper introduces a new software concept - the intelligent being - that delivers the suitability for integration. Furthermore, the paper addresses which components and services can be developed in this manner. The discussion argues that intelligent products and intelligent beings are a natural match. Furthermore, intelligent beings are attractive components for the development of a system foundation since they are capable of surviving changes and confrontation with other systems unscathed.

Concerning work-by-others, McFarlane (2003) discusses the application of RFID technology in intelligent manufacturing control. His team has been generalizing the concept of an Intelligent Product to cover intelligent resources (Wong 2002). To shed some light on this, it is useful to understand how intelligent products correspond to three main categories depending on which part in the world-of-interest they reflect and/or correspond to:

1. A product type (non-material, virtual, knowledge only)

2. A product instantiation (the activity of producing a product instance)

3. A product instance.

Each of those categories should have its own intelligent being/product. The main motivation to demand separate intelligent products is similar to the motivation to cleanly separate intelligent being from intelligent agents: their user communities and markets are different. Having separate intelligent products increases critical user mass versus the software component complexity.

The first two categories are self-explaining. An intelligent product types is knowledgeable about a product design and process plans. An intelligent product instantiation is knowledgeable about the activity that is producing a product instance (i.e. production). The intelligent product instance covers the remainder of the life cycle for the result of a successful product instantiation.

An interesting category of intelligent product instances are intelligent resources. Indeed, production equipment and other production means are themselves products. In this manner, intelligent resources are a subcategory of intelligent product instances (in the usage phase of their life cycle). Such a unifying view on intelligent components/products is likely to benefit from cross-fertilization. For instance, emulation services, commonly provided for production equipment, may become more commonplace and become available on ordinary products. This opens perspectives of virtual eco-audits, virtual assessment of accessibility by users with special requirements, etc.

Overall, the analysis and discussion on how to design and structure the internet of things has only just started. This paper demonstrates that significant innovation opportunities are present and that fundamental rethinking may yield significant benefits.

\subsection{Acknowledgments}

This paper presents work funded by the Research Fund of the K.U.Leuven - Concerted Research Action on Autonomic Computing for Distributed Production Systems. 


\section{REFERENCES}

1. Jackson, M. Software requirements and specifications. Addison-Wesley, 1995.

2. McFarlane, D., Sarma, S., Chirn, JL, Wong, CY, Ashton, K. Auto ID systems and intelligent manufacturing control. Engineering Applications of Artificial Intelligence Jun 2003; 16 (4): 365-376.

3. Parunak, HVD, Brueckener, S., Weyns, D. Holvoet, T., Verstraete, P., Valckenaers, P., E Pluribus Unum: PolyAgent and Delegate MAS Architectures, Eight International Workshop on Multi-Agent Based Simulation at AAMAS 2007, Honululu, Hawaii, 15 May 2007, forthcoming in the proceedings

4. Valckenaers, P., Van Brussel, H., Hadeli, Bochmann, O., Saint Germain, B., Zamfirescu, C. On the Design of Emergent Systems: an Investigation of Integration and Interoperability Issues. Engineering Applications of Artificial Intelligence 2003; 16: 377-393.

5. Valckenaers, P. and Van Brussel, H. Holonic Manufacturing Execution Systems. CIRP Annals Manufacturing Technology 2005; 54/1: 427-432.

6. Valckenaers P., Sauter J., Sierra C., Rodriguez-Aguilar J.A. Applications and environments for multi-agent systems. Autonomous Agents and Multi-Agent Systems Feb 2007; 14 (1): 61-85

7. Wong, C.Y., McFarlane, D., Zaharudin, A., Agarawal, V. The intelligent product driven supply chain. Proceedings of IEEE Systems Man and Cybernetics, Hammamet, Tunisia, 2002. 